\title{
Optimal VGOS telescope location for the estimation of Earth orientation parameters
}

\section{Other Conference Item}

Author(s):

Schartner, Matthias (i); Böhm, Johannes; Nothnagel, Axel

Publication date:

2020-05-07

Permanent link:

https://doi.org/10.3929/ethz-b-000459549

Rights / license:

Creative Commons Attribution 4.0 International

Originally published in:

EGUsphere, https://doi.org/10.5194/egusphere-egu2020-13484 
EGU2020-13484

https://doi.org/10.5194/egusphere-egu2020-13484

EGU General Assembly 2020

(c) Author(s) 2021. This work is distributed under

the Creative Commons Attribution 4.0 License.

\section{Optimal VGOS telescope location for the estimation of Earth orientation parameters}

Matthias Schartner, Johannes Böhm, and Axel Nothnagel

TU Wien, Higher Geodesy, Department of Geodesy and Geoinformation, Wien, Austria

(matthias.schartner@geo.tuwien.ac.at)

In this investigation, we identify optimal locations for VGOS radio telescopes to estimate Earth orientation parameters (EOP) with a new method based on bulk schedule generation and largescale Monte-Carlo simulations. Thereby, we focus on a high number of simulations and a proper consideration of scheduling to minimize these undesired error sources.

The location of the telescope is varied over 477 possible locations, homogeneously distributed over land areas on the globe. The antenna is added to a fixed network of 6,12 and 18 existing and upcoming VGOS stations. The optimal location is defined through the minimal resulting repeatabilities of the simulated EOP. In this study, a special focus was laid on the generation of high-quality observing plans to minimize the effects of scheduling combined with a high number of simulations to minimize their randomness. To remove the unintended effects caused by scheduling over 93 thousand schedules were iteratively generated. Each schedule is further simulated 1000 times leading to over 5 trillion simulated and analyzed observations. Besides showing our results for the best telescope location, we will highlight how scheduling and the number of simulations affects the repeatability of the estimated EOP. This will help further simulation studies to improve their results.

The optimal telescope location depends on the EOP of interest and the existing network. For simple network geometries, such as the 6 station network which consists of antennas in Europe and North America, the importance of east-west baselines can be seen for the determination of dUT1 while the importance of north-south baselines can be seen for the determination of polar motion and nutation. For more complex network geometries and an increasing number of VGOS stations, the lack of southern stations becomes more prominent. For the 12 and 18 station network, the location of an additional antenna in south America can significantly improve the accuracy of the EOP by up to $60 \%$. 